\title{
The Relationship Between Ankle Muscles and An EMG-Based Physically Interactive Game
}

\author{
Yu-Min Ko' ${ }^{1}$ Seol Park², Ho-Cheol Lee ${ }^{3}$, Chang-Hun Lim4, Ji-Won Park ${ }^{5}$ \\ 'Department of Physical Therapy, Gangneung Yeongdong College, Gangneung; ${ }^{2}$ Department of Physical Therapy, College of Medical Science, \\ Catholic University of Daegu, Daegu; ${ }^{3}$ Faculty of Mechanical and Automotive Engineering, College of Engineering Catholic University of Daegu, \\ Gyeongsan; ${ }^{4}$ Department of Physical Therapy, Gangneung Yeongdong College, Gangneung; ${ }^{5}$ Department of Physical Therapy, College of Medical \\ Science, Catholic University of Daegu, Daegu, Korea
}

Purpose: This study was to identify the relationship between the game score and muscle strength in order to elucidate whether the obtained score for the dorsiflexor and plantar flexor muscles in the ankle joint using an EMG-based interactive game system can reflect muscle strength as measured conventionally.

Methods: Forty adults were enrolled in the present study. They had no congenital deformities, and no neurological or orthopedic disorders in the 6 months prior to the start of the study. The Biodex were used to measure the isokinetic concentric maximal strength of the plantar flexor and dorsiflexor muscles in the ankle joint. EMG electrodes were attached to the tibialis anterior and gastrocnemius.

Results: (1) There was a positive relationship between the obtained game score by the plantar flexor (SPF) and muscle strength of the plantar flexor (tPF) and dorsiflexor (tDF). In addition, the tPF affected the sPF, but the tDF did not. Thus, the higher the tPF, the higher the sPF. (2) There was no relationship between the obtained game score of dorsiflexor (SDF) and tPF or tDF. In addition, neither the tDF or tPF affected the SDF.

Conclusion: The game score had a relationship with muscle strength, which is related to ankle instability and re-impairment. Thus we suggest that this game system can be used to predict the degree of weakness of muscle strength.

Keywords: Muscle strength, Dorsiflexor, Plantar flexor, Interactive game, Game score

\section{INTRODUCTION}

Rehabilitation is any treatment performed to recover physical, mental, or social ability when the quality of life has been decreased by disability, temporary trauma, pain, or disorders. ${ }^{1,2}$ One of the typical treatments is therapeutic exercise, where a therapist helps to recover or maintain a patient's motor function by moving the patient's joint passively, or by educating and assisting the patient's gait. This therapeutic exercise is needed for a long period of time for treatment and endurance, and the patient's potential power of recovery and active will to participate are key factors. ${ }^{3}$ In particular, how cooperative a patient is in active participation is vital to the level of improvement during rehabilitation and the maintenance of rehabilitation activities, and becomes a new power in life without specific treatment,

Received Nov 11, 2015 Revised Dec 22, 2015

Accepted Dec 24, 2015

Corresponding author Ji-Won Park

E-mail mylovept@hanmail.net enhancing personal relations and independence. ${ }^{3,4}$ Jang \& Yoon ${ }^{4}$ showed that rehabilitation will can have a huge effect on treatment success

Kang $^{5}$ developed an EMG-based physically interactive game system to improve rehabilitation environments as the necessity to improve monotonous or boring long-lasting rehabilitation had been recognized, as the application of this game system using EMG biofeedback was shown to improve a patient's active will for participation, and to increase patient satisfaction. EMG biofeedback from Kang's study is used widely in medical science, rehabilitation medicine, human engineering, and sports science, due to the fact that it can be an important factor to improve the efficiency of therapeutic exercise by checking a patient's muscles and applying patient training $^{6,7}$ Biofeedback training can identify real-time vital signs seen by

Copylight (C2015 The Korea Society of Physical Therapy

This is an Open Access article distribute under the terms of the Creative Commons Attribution Non-commercial License (Http:// creativecommons.org/license/by-nc/3.o.) which permits unrestricted non-commercial use, distribution, and reproduction in any medium, provided the original work is properly cited. 
the patient themselves, thus it can enhance small increases in achievement towards goals, and rapidly facilitate the process of rehabilitation. ${ }^{8-10}$ Biofeedback training using EMG is the best method to recover injured motor function of the upper limb. ${ }^{11}$ This application of a game system using EMG biofeedback can induce correct movement through the use of EMG signals relating to paralyzed or weakened muscles, and improve motor performance by facilitating motor learning using vital signs that are provided in real-time. ${ }^{8} \mathrm{~A}$ game system using EMG biofeedback may need proper timing of contraction and relaxation of muscles, control of neural networks of neuromuscular system, and accurate movement in order to control a game character through inducing muscle contraction, while general EMG biofeedback system provides simple visual information using numbers or graph about muscle activities or auditory information using beeps when muscle activity is over than threshold. Moreover, the application of a game system can enhance a patient's active will for participation and increase satisfaction of treatment by improving monotonous or boring rehabilitation environments. ${ }^{6}$

However, there is a question of whether the application of an EMG-based physically interactive game system can be used as rehabilitation. Due to the fact that Kang's study confirmed patients' subjective satisfaction regarding the application of a game system through questionnaires, therefore he could not provide the answer as to whether this system could be an alternative to other types of rehabilitation. Thus, to suggest a game system as an alternative to rehabilitation, an investigation was needed in order to recognize if the application of a game system could improve a patient's kinetic characteristics. Prior to this, it was necessary to identify whether the game system could replace existing measurement equipment by the verification that there is a significant relationship between the score obtained by the game system and the muscle strength measured with existing measurement equipment.

Chronic instability of the ankle joint is progressed by injury such as an ankle sprain, and is caused by weak muscle strength of the tibialis anterior and peroneus longus, as well as decreased proprioception. ${ }^{12-14}$ The present study aims to analyze the relationship between the game score and muscle strength in order to elucidate whether the obtained score for the dorsiflexor and plantar flexor muscles in the ankle joint using an EMG-based physically interactive game system can reflect muscle strength as measured conventionally.

\section{METHODS}

\section{Subjects}

40 subjects (mean age $22.8 \pm 2.3$ years old, height $166.9 \pm 7.6 \mathrm{~cm}$, body weight $56.9 \pm 9.8 \mathrm{~kg}$, and BMI $20.3 \pm 2.2 \%$ ) were enrolled in the present study. They had no congenital deformities, and no neurological or orthopedic disorders in the 6 months prior to the start of the study. The subjects' general characteristics were measured including their height and body weight before experiments. 12 subjects were male with a mean age of $25.1 \pm 2.0$ years old, a height of $176.3 \pm 4.1 \mathrm{~cm}$, a body weight of $69.1 \pm 6.7 \mathrm{~kg}$, and a BMI of $22.3 \pm$ $2.2 \% .28$ subjects were female with a mean age of $21.8 \pm 1.7$ years old, a height of $162.8 \pm 4.5 \mathrm{~cm}$, a body weight of $51.6 \pm 5.0 \mathrm{~kg}$, and a BMI of $19.5 \pm 1.5 \%$. The subjects agreed to participate in the study following an explanation of the experiments.

\section{Experimental methods}

\section{1) Measurements}

\section{(1) Biodex system}

The Biodex system 4 Pro Dynamometer and the Biodex Advantage Software Package (Biodex Medical System Inc., Shirley, USA) were used to measure the isokinetic concentric maximal strength of the plantar flexor and dorsiflexor muscles in the ankle joint. The lower leg was on a support with a height of $40 \mathrm{~cm}$, and fixed using a strap in a full lying posture on a chair. The axis of the joint corresponded to the axis of the dynamometer. The opposite thigh, which was not being measured, was fixed using a strap in the $90^{\circ} \mathrm{knee}$ flexion position, with the foot on a support. Isokinetic concentric maximal strength was calculated as the peak torque divided by the body weight, and the angular velocity was $60 \% \mathrm{sec}$. Measurements were taken 5 times, and the mean value was used for analysis. Subjects were encouraged by bulbar command consistently.

\section{(2) EMG-based biofeedback game system}

EMG electrodes were attached to the tibialis anterior and gastrocnemius to obtain the game score using the EMG-based biofeedback game system QEMG-4XL (Laxtha, Korea). Electrodes on the tibialis anterior (ankle dorsiflexor) were attached $2 \mathrm{~cm}$ laterally on the tibial line, and electrodes on the gastrocnemius (ankle plantar flexor) were attached $2 \mathrm{~cm}$ inferiorly and medially from the center line of the popliteal fossa. ${ }^{15}$ Following attachment of the EMG electrodes, 
individual muscle strengths were calibrated to consider individual variations. The game was then performed 3 times, and the mean score was used for analysis. The relationship between the mean game score and the peak torque/body weight was analyzed.

The game character could be moved up or down according to the EMG signal obtained by each muscle contraction, and was designed so that various types of fish were to be passed from the right to left hand side of the screen. When the game character made contact with a passing fish, a point was acquired, whereas when the character could not contact a fish three times, the game was over.

\section{2) Statistical Analysis}

SPSS version 18.0 for windows was used for statistical analysis. Pearson correlation was used to elucidate the relationship between the game score and muscle strength, and regression was used to elucidate whether muscle strength had an effect on the game score. The significance level was set at 0.05 .

\section{RESULTS}

(1) The relationship between the game score and muscle strength of the plantar flexor.

There was a positive relationship between the obtained game score by the plantar flexor (sPF) and muscle strength of the plantar flexor (tPF) and dorsiflexor (tDF) (Table 1).

In addition, the tPF affected the sPF, but the tDF did not. Thus, the higher the $\mathrm{PF}$, the higher the sPF (Table 2).

(2) The relationship between the game score and muscle strength of the dorsiflexor.

There was no relationship between the obtained game score by the dorsiflexor (sDF) and tPF or tDF (Table 1). In addition, neither the tPF or tDF affected the sDF (Table 3).

Table 1. The correlation between peak torque/body weight and game score of ankle plantar flexor and dorsiflexor

\begin{tabular}{lccc}
\hline & tPF & tDF & sPF \\
value $(\mathrm{M} \pm \mathrm{SD})$ & $27.52 \pm 22.08$ & $17.58 \pm 20.26$ & $192.37 \pm 45.53$ \\
tPF & 1 & $0.65^{*}$ & $0.58^{*}$ \\
tDF & & 1 & 0.05 \\
SPF & & & 0.28 \\
\hline
\end{tabular}

tPF: Peak torque/body weight of plantar flexor in ankle joint, tDF: Peak torque/body weight of dorsiflexor in ankle joint, sPF: Game score of plantar flexor in ankle joint, SDF: Game score of dorsiflexor in ankle joint.

* Statistically significant at the level of $p<0.01$.

Table 2. The results of regression between game score and peak torque/body weight of plantar flexor in ankle joint

\begin{tabular}{|c|c|c|c|c|c|c|c|}
\hline & & $\mathrm{R} 2$ & $B(\beta)$ & SE & $\mathrm{F}$ & $t$ & $p$ \\
\hline \multirow[t]{2}{*}{ tPF } & Constant & & 225.34 & 9.82 & & 22.96 & 0.00 \\
\hline & $\mathrm{tPF}$ & 0.34 & $1.20(0.58)$ & 0.28 & 18.35 & 4.28 & $0.00^{*}$ \\
\hline \multirow[t]{2}{*}{ tDF } & Constant & & 197.32 & 16.90 & & 11.67 & 0.00 \\
\hline & $\mathrm{tDF}$ & 0.00 & $0.28(0.05)$ & 0.85 & 0.11 & 0.33 & 0.75 \\
\hline \multirow[t]{2}{*}{ sDF } & Constant & & 46.42 & 79.25 & & 0.59 & 0.56 \\
\hline & sDF & 0.08 & $0.66(0.30)$ & 0.36 & 3.42 & 1.85 & 0.07 \\
\hline
\end{tabular}

tPF: Peak torque/body weight of plantar flexor in ankle joint, tDF: Peak torque/body weight of dorsiflexor in ankle joint, sDF: Game score of dorsiflexor in ankle joint. *Statistically significant at the level of $p<0.01$.

Table 3. The results of regression between game score and peak torque/body weight of dorsiflexor in ankle joint

\begin{tabular}{|c|c|c|c|c|c|c|c|}
\hline & & $\mathrm{R} 2$ & $B(\beta)$ & SE & $\mathrm{F}$ & $\mathrm{t}$ & $\mathrm{p}$ \\
\hline \multirow[t]{2}{*}{$\mathrm{tPF}$} & Constant & & 227.46 & 5.16 & & 44.06 & 0.00 \\
\hline & tPF & 0.08 & $0.25(0.28)$ & 0.15 & 2.93 & 1.71 & 0.10 \\
\hline \multirow[t]{2}{*}{$\mathrm{tDF}$} & Constant & & 230.78 & 7.29 & & 31.67 & 0.00 \\
\hline & tDF & 0.06 & 0.58 & 0.37 & 2.46 & 1.57 & 0.13 \\
\hline \multirow[t]{2}{*}{ sDF } & Constant & & 195.31 & 14.00 & & 13.95 & 0.00 \\
\hline & sDF & 0.09 & $0.13(0.30)$ & 0.07 & 3.42 & 1.85 & 0.07 \\
\hline
\end{tabular}

tPF: Peak torque/body weight of plantar flexor in ankle joint, tDF: Peak torque/body weight of dorsiflexor in ankle joint, sPF: Game score of plantar flexor in ankle joint. * Statistically significant at the level of $p<0.01$. 


\section{DISCUSSION}

The present study examined whether the game score using an EMG-based physically interactive game system, which was developed to overcome monotonous and boring long-lasting rehabilitation training and to induce patients' active participation, is related to muscle strength as measured by traditional equipment. ${ }^{16,17}$

The peak torque/body weight of the plantar flexor was greater than the peak torque/body weight of the dorsiflexor. This is consistent with Ko's study in which it was reported that plantar flexor strength was greater than dorsiflexor strength when measuring the muscle strength balance index in the ankle joints of healthy young women. ${ }^{18}$ The gastrocnemius as the plantar flexor may be stronger than the tibialis anterior as the dorsiflexor, due to the fact that the gastrocnemius has a larger motor unit, and a very strong power to lift body weight, such as lifting heels against body weight and gravity. The center of gravity on the body is just in front of the ankle joint on standing. ${ }^{19}$ The dorsiflexor lifts only the weight of the foot, but the plantar flexor lifts body weight, thus the gastrocnemius should have a stronger power than the tibialis anterior. This is supported by the isokinetic concentric maximal strength/body weight in the present study.

Although the maximal strength of the plantar flexor was greater than that of the dorsiflexor, the obtained game score by the dorsiflexor $(\mathrm{sDF})$ was higher than that of the plantar flexor. The dorsiflexor has a smaller motor unit because its size and isokinetic strength are smaller than that of the plantar flexor. Delicate control of the ankle joint was needed for the game system, and the dorsiflexor, which has a smaller motor unit, controls detailed movement of the ankle joint, thus the sDF was higher.

There was a positive relationship between the peak torque/body weight of the plantar flexor and the dorsiflexor, meaning that the stronger the plantar flexor strength, the stronger the dorsiflexor strength.

On the other hand, the obtained game score by the plantar flexor $(\mathrm{sPF})$ was affected by the plantar flexor strength (tPF). The greater the $\mathrm{PPF}$, the higher the sPF. Individuals with a lower sPF may have weak tPF. However, there was no relationship found between the sPF and dorsiflexor strength (tDF).

The sDF was not affected by the tPF or tDF. Subjects had a higher sDF unlike the sPF, meaning that there was no difference in sDF with respect to muscle strength. The game required more delicate control of the ankle joint than greater strength, thus the dorsiflexor, which has a smaller motor unit, controls detailed movements of the ankle joint. The present study measured the dorsiflexor in healthy young adults, and they did not have any severe muscle injury or pain in the ankle joint, therefore the sDF was higher in all subjects. Joint instability or pain in the ankle joint is related to poor strength of the dorsiflexor and decreased sense of position,,$^{20}$ and it can be suggested that joint instability or pain affects the game score. Thus, measuring the game score can predict poor strength of the dorsiflexor or poor sense of position. On the other hand, in order to predict strength in a healthy ankle joint or proprioception without injury or pain, application of the game score using the plantar flexor can have a more accurate prediction than using the dorsiflexor.

As a result, $\mathrm{tPF}$ was greater than $\mathrm{tDF}$, however the $\mathrm{sDF}$ was higher than sPF. The sPF was affected by tPF, but the sDF was not affected by the strength of either the plantar flexor or the dorsiflexor.

Chronic instability of the ankle joint is progressed by injury such as an ankle sprain, and is caused by weak muscle strength of the tibialis anterior and peroneus longus, as well as decreased proprioception. ${ }^{12-14}$ If strength of the ankle joint and proprioception maintain a normal function, ankle stability is maintained, and re-impairment can be prevented. ${ }^{21}$ The present study shows that the game score measured using an EMG-based physically interactive game system had a relationship with muscle strength, which is related to ankle instability and re-impairment. Thus we suggest that this game system can be used to predict the degree of weakness of muscle strength.

The limitations in this study were as following: first, there were no control groups, because this study investigated the relationship between the degree of the muscle strength and game score. Second, the game system was not used as an intervention.

In future studies, examination of the relationship between the game score obtained with this game system and proprioception or muscle balance index that causes instability needs to be carried out. Moreover, verification that this game system has therapeutic effects as rehabilitation equipment, by experiments conducted in healthy individuals and patients, is required.

\section{REFERENCES}

1. Lee DY. A study on the vocational rehabilitation facilities: Focusing on 
the current situations, conditions and administrative efficiency. Korean Journal of Physical Multiple \& Health Disabilities. 2010;53(1):1-24.

2. Choi YS. A Review of rehabilitation sports for the disabled. Korean Academic Society of Rehabilitation Nursing. 2009;12(1):47-54.

3. Lee DY, Lee MS, Na BJ et al. Related factors of the motivation for rehabilitation in stroke patients. Korean Research Society of Physical Therapy. 2006;13(1):16-23.

4. Jang JY, Yoon YS. A Study on serious game design for rehabilitation training of sroke patients. International Jour of KSCG. 2008;15:151-9.

5. Kang JH, Kim JY, Park JH et al. Implementation of EMG acquisition system and application for the improvement of rehabilitation treatment environment. KITE Journal of Electronics Engineering. 2010;6:1383-6.

6. Kim GS, Kang JH, Moon CS et al. The Development of interactive wireless-game device based on multi-channel electromyography for rehabilitation in patients with hemiplegia. Journal of Korea Game Society. 2011;11(6):171-80.

7. Eleanor C. Cram's introduction to surface electromyography. 2nd Edition. Burlington, Jones \& Bartlett Learning, 2005:5-7.

8. Mulder T, Hulstyn W. Sensory feedback therapy and theoretical knowledge of motor control and learning. Am J Phys Med. 1984;63(5):226-44.

9. Park SK, Kang JY. Effects of EMG-biofeedback training on functional ability and Q-angle in patellofemoral pain syndrome. J Kor Phys Ther. 2014;26(2):68-73.

10. Park JJ, So HJ, Shin WS. Effects of gluteus medius strengthening training using pressure biofeedback unit for muscle function and balance in stroke patients. J Kor Phys Ther. 2015;27(4):221-7.

11. Van DH, Jannink MJ, Hermens HJ. Effect of augmented feedback on motor function of the affected upper extremity in rehabilitation patients: A systematic review of randomized controlled trials. J Rehabil Med. 2005;
37(4):202-11.

12. Mattacola CG, Dwyer MK. Rehabilitation of the ankle after acute sprain or chronic instability. J Athl Train. 2002;37(4):413-29.

13. Osborne MD, Chou LS, Laskowski ER et al. The effect of ankle disk training on muscle reaction time in subjects with a history ankle sprain. Am J sports med. 2001;29(5):627-32.

14. Kaminski TW, Hartsell HD. Factors contributing to chronic ankle instability: A Strength perspective. J Athl Train. 2002;37(4):394-405.

15. Schulthies SS, Ricard MD, Alexander KJ et al. An electromyographic investigation of 4 elastic-tubing closed kinetic chain exercises after anterior cruciate ligament reconstruction. J Athl Train. 1998;33(4):328-35.

16. Kim YK, Song JC, Choi JW et al. Functional electric stimulation-assisted biofeedback therapy system for chronic hemiplegic upper extremity function. J Kor Phys Ther. 2012;24(6):409-13.

17. Park SK, Kim JH. Effects of EMG-biofeedback training on total knee replacement patients' lower extremity muscle activity and balance. J Kor Phys Ther. 2013;25(2):81-7.

18. Ko YM, Jung MS, Park JW. The Relationship between strength balance and joint position sense related to ankle joint in healthy women. J Kor Phys Ther. 2011;23(2):23-9.

19. Neumann DA. Kinesiology of the musculoskeletal system: Foundations for rehabilitation, 2nd Edition. Elsevier Science Health Science Division. 2010.

20. Willems T, Witvrouw E, Verstuyft J et al. Proprioception and muscle strength in subjects with a history of ankle sprains and chronic instability. J Athl Train. 2002;37(4):487-93.

21. Denegar CR, Miller SJ. Can chronic ankle instability be prevented? Rethinking management of lateral ankle sprains. J Athl Train. 2002; 37(4):430-5. 